\begin{tabular}{|c|c|}
\hline \multirow{3}{*}{ 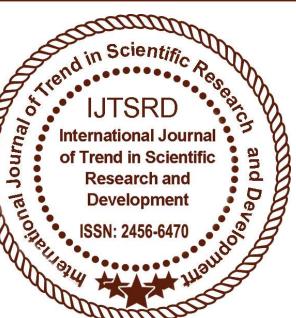 } & $\begin{array}{l}\text { International Journal of Trend in Scientific } \\
\text { Research and Development (IJTSRD) }\end{array}$ \\
\hline & International Open Access Journal \\
\hline & ISSN No: $2456-6470$ | www.ijtsrd.com | Volume - 2 | Issue -4 \\
\hline
\end{tabular}

\title{
Situation invariant Face Recognition using Neural Networks
}

\author{
Prof. Suryawanshi Rupali Vithalrao \\ Assistant Professor, Department of Electrical \\ Engineering, BMIT, Solapur, Maharashtra, India
}

\author{
Prof. Kazi Kutubuddin Sayyad Liyakat \\ Associate Professor, Department of Electronics and \\ Telecommunication Engineering, BMIT, \\ Solapur, Maharashtra, India
}

\begin{abstract}
Here we consider human face as biometric. Original method of feature extraction from image data is introduced using feed forward Neural Network (multilayer perceptron) and PCA (principal component analysis). This method is used in human face recognition system and results are compared to face recognition system using PCA directly, to a system with direct classification of input images by NN a network, and to a system using PCA as a feature extractor and $\mathbf{N N}$ network in the role of
\end{abstract} classifier.

Keywords:- PCA, NN, EPOCH, Eigenvalue, Eigen Vector

\section{Principal Component Analysis: -}

Principal component analysis PCA [1] is a standard statistical method used for feature extraction. It transforms input data represented by a random vector $\mathrm{x}=\left[\mathbf{x}_{\mathbf{0}}, \mathbf{x}_{\mathrm{b}}, \boldsymbol{x}_{2}, \ldots, x_{p-1}\right]^{T}, \mathrm{E}[\mathrm{x}]=0$ with correlation matrix $\mathbf{R}_{\mathbf{X}}=\mathrm{E}\left[\mathrm{XX}^{\mathrm{T}}\right]=\mathrm{R}^{\mathrm{T}} \mathrm{x}$ to a set of coefficients (principal components)

$$
a_{j}=\mathbf{u}_{j}^{T} \mathbf{x}=\mathbf{x}^{T} \mathbf{u}_{j}, j=0,1, \ldots, p-1
$$

represented by the vector $\mathrm{a}=[\mathrm{ao}, \mathrm{a} 1, \mathrm{a} 2, \ldots, \mathrm{ap}-1]^{\mathrm{T}}$.

Unit vectors $u \quad{ }_{j}=\left[\begin{array}{llll}\boldsymbol{u}_{j}, \boldsymbol{u}_{j}, \boldsymbol{u}_{j, 2 . .} & , \boldsymbol{u} & j p . I]^{T}\end{array}\right.$ $\left(\|\mathbf{u}\|=\sqrt{\mathbf{u}^{T} \mathbf{u}}=1\right)$ forms the matrix $\mathrm{U}=[\mathbf{u o}$, $\mathbf{u 1}, \mathbf{u} 2$, ..u p-l ] and they are eigenvectors of the correlation matrix $\mathbf{R}_{\mathbf{X}}$, associated with the eigenvalues

$\lambda_{0}, \lambda_{1}, \ldots, \lambda_{p-1}$, where $\lambda_{0}>\lambda_{1}>\ldots>\lambda_{p-1}$ and $\lambda_{0}=\lambda_{M U X}$.
The most important eigenvectors are those corresponding to largest eigenvalues of $\mathrm{R}_{\mathrm{X}}$

The representation of input data (analysis, forward transform) is defined by

$$
\mathbf{a}=\mathbf{x}^{T} \mathbf{U}=\mathbf{U}^{T} \mathbf{x}
$$

and synthesis (inverse transform) is represented by

$$
\mathbf{x}=\mathbf{U} \mathbf{a}=\sum_{j=0}^{p-1} a_{j} \mathbf{u}_{j}
$$

It is possible to represent input data by a reduced number of principal components (dimensionality reduction). The transform uses eigenvectors corresponding to largest eigen values of $\mathrm{R}_{\mathrm{X}}$, and those corresponding to small eigen values are discarded

$$
\mathbf{x}^{\prime}=\sum_{j=0}^{m-1} a_{j} \mathbf{u}_{j}, m<p
$$

Then vector $x^{\prime}$ is an approximation of $x$, while

$$
\lambda_{0}>\lambda_{1}>\ldots>\lambda_{m-1}>\lambda_{m}>\ldots>\lambda_{p-1} .
$$

\section{Feed Forward Multilayer Neural Network:-}

Basic multilayer NN building unit is a model of artificial neuron. This unit computes the weighted sum of the inputs plus the threshold weight and passes 
these sums through the activation function (usually sigmoid) [1]:

$$
\begin{aligned}
& v_{j}=\theta_{j}+\sum_{i=1}^{p} w_{j i} x_{i}=\sum_{j=0}^{p} w_{j i} x_{i} \\
& y_{j}=\varphi_{j}\left(v_{j}\right)
\end{aligned}
$$

Where $\mathrm{v}_{\mathrm{j}}$ is linear combination of inputs $\mathrm{x} 1, \mathrm{x} 2, \ldots, \mathrm{xp}$ of neuron $\mathrm{j}, \boldsymbol{w}_{\boldsymbol{j} \boldsymbol{o}}=\theta_{\boldsymbol{j}}$ is threshold weight connected to special input xo $=-1, \boldsymbol{y}_{\boldsymbol{j}}$ is the output of neuron $\mathrm{j}$ and $\psi_{j}($.$) is its activation function. Herein we use special$ form of sigmoidal (non-constant, bounded, and monotone-increasing) activation function -logistic function

$$
y_{j}=\frac{1}{1+\exp \left(-v_{j}\right)}
$$

In a multilayer perceptron, the outputs of the units in one layer form the inputs to the next layer. The weights of the network are usually computed by training the network using the back-propagation (BP) algorithm. A multilayer perceptron represents nested sigmoidal scheme [I], its form for single output neuron is

$$
F(\mathbf{x}, \mathbf{w})=\varphi\left(\sum_{j} w_{o j} \varphi\left(\sum_{k} w_{j k} \varphi\left(\ldots \varphi\left(\sum_{i} w_{l i} x_{i}\right) \ldots\right)\right)\right)
$$

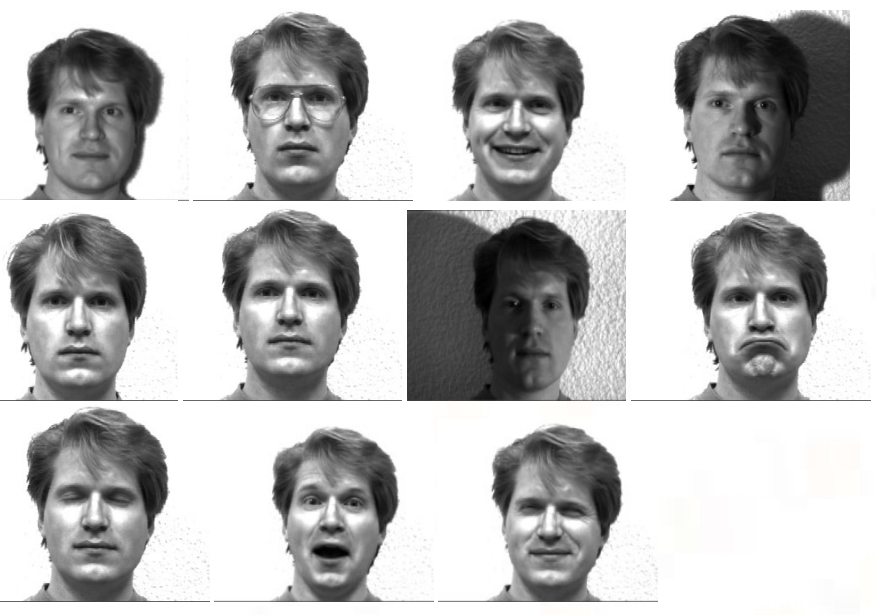

Figure: - The Subject with different eleven poses used in project as per Yale database

\section{RECOGNITION METHOD:}

PCA applied directly to face images with Euclidian distance as a classification measure, shown in figure bellow. The correlation matrix was computed from 8 training faces and for classification first 8 eigenvectors of the correlation matrix are used 93.54 $\%$ of test faces was recognized successfully. This result corresponds to method as shown in figure bellow.

$$
\text { Input } \underset{x_{2}}{x_{1} \rightarrow x_{1}} \text { PCA }
$$

K-nearest neighbor algorithm: -In pattern where $\varphi(\cdot)$ is a sigmoidal activation function, $\boldsymbol{w}_{\boldsymbol{o j}}$ is the synaptic weight from neuron $\mathrm{j}$ in the last hidden layer to the single output neuron $\mathbf{0}$, and so on for the other synaptic weights, $x i$ is the $i^{\text {th }}$ element of the input vector $\mathbf{x}$. The weight vector $\mathrm{w}$ denotes the entire set of synaptic weights ordered by layer, then neurons in a layer, and then number in a neuron.

\section{FACE DATABASE}

We use the face database from Yale database, which consists of face images of 15 people (shown in Fig. I), 11 for each person under various passions \& scale. It means, total number of face images is 165 . Each image is $240 \times 320$ pixels, eight-bit grayscale. An example of different face images (patters) belonging to the same class is shown in Figure bellow.

recognition, the k-nearest neighbor algorithm (k-NN) is a method for classifying objects based on closest training examples in the feature space. $\mathrm{k}-\mathrm{NN}$ is a type of instance-based learning, or lazy learning where the function is only approximated locally and all computation is deferred until classification. It can also be used for regression. The best choice of $\mathrm{k}$ depends upon the data; generally, larger values of $k$ reduce the effect of noise on the classification, but make boundaries between classes less distinct. A good $\mathrm{k}$ can be selected by various heuristic techniques, for example, cross-validation. The special case where the class is predicted to be the class of the closest training sample (i.e. when $\mathrm{k}=1$ ) is called the nearest neighbor algorithm. The accuracy of the k-NN algorithm can be severely degraded by the presence of noisy or irrelevant features, or if the feature scales are not consistent with their importance. Much research effort has been put into selecting or scaling features to improve classification. A particularly popular 
approach is the use of evolutionary algorithms to optimize feature scaling. Another popular approach is to scale features by the mutual information of the training data with the training classes.

The nearest neighbor algorithm has some strong consistency results. As the amount of data approaches infinity, the algorithm is guaranteed to yield an error rate no worse than twice the Bayes error rate (the minimum achievable error rate given the distribution of the data). $k$-nearest neighbor is guaranteed to approach the Bayes error rate, for some value of $k$ (where $k$ increases as a function of the number of data points). The $k$-NN algorithm can also be adapted for use in estimating continuous variables. One such implementation uses an inverse distance weighted average of the $k$-nearest multivariate neighbors. This algorithm functions as follows:

1. Compute Euclidean distance from target plot to those that were sampled.

2. Order samples taking for account calculated distances.

3. Choose heuristically optimal $k$ nearest neighbor based on RMSE done by cross validation technique.

4. Calculate an inverse distance weighted average with the $k$-nearest multivariate neighbors.

Epoch (astronomy): -In astronomy, an epoch (or sometimes epochal moment) is a moment in time for which celestial coordinates or orbital elements are specified. In the case of celestial coordinates, and with modern technology, the position at other times can be computed by taking into account precession and proper motion. In the case of orbital elements, it is necessary to take account of perturbation by other bodies in order to calculate the orbital elements for a different time. The currently used standard epoch is J2000.0, which is January 1, 2000 at 12:00 TT. The prefix "J" indicates that it is a Julian epoch. The previous standard epoch was B1950.0, with the prefix "B" indicating it was a Besselian epoch.

\section{CONCLUSION:}

The main limitation of the current system is that it only detects upright faces looking at the camera. Separate versions of the system could be trained for each Lightening condition, and the results could be combined using arbitration methods similar to those presented here. Preliminary work in this area indicates that detecting profiles views of faces is more difficult than detecting frontal views, because they have fewer stable features and because the input window will contain more background pixels. We have also applied the same algorithm for the detection of car tires and human eyes, although more work is needed. Even within the domain of detecting frontal views of faces, more work remains. When an image sequence is available, temporal coherence can focus attention on particular portions of the images. As a lighting condition changes, as per the its location in one frame is a strong predictor of its location in next frame. Standard tracking methods, as well as expectationbased methods, can be applied to focus the detector's attention..

\section{REFERENCE:}

1. Face Recognition Methods Based on Principal Component Analysis and Feed-forward Neural Networks by: - Milo5 Oravec, Jarmila PavloviEov6, IEEE paper number- 0-7803-83S91/04, C2004 IEEE

2. Face Recognition by Using Neural Network Classifiers Based on PCA and LDA by: -ByungJoo Oh,

3. Wavelets and Neural Networks Based Face Recognition System by: - M. R. M. Rizk, S.M., O. Said and R. El-Sayed IEEE paper number - 07803-8294-3104/ C2004 IEEE

4. High-Speed Face Recognition Based on Discrete Cosine Transform and RBF Neural Networks by: Meng Joo Er, Member, IEEE transactions on neural networks, vol. 16, No. 3, May 2005

5. Book on "Neural Networks for Pattern recognition" by Christopher M. Bishop Published by Oxford University Press.

6. National Conference on Emerging Trends in Electronics \& Telecommunication@ @SCOE, Pune@ NCET-2007 IEEE sponsored program, on $16^{\text {th }} \& 17^{\text {th }}$ April -07 on topic "Face Recognition Based on Neural Network". The Article number 2 in ANN group Session-III on page no.-132-135.

7. National Conference on Emerging Trends in Signal Processing \& Communication @ MAE, Alandi, Pune in ETSPC-07 @ 27 $7^{\text {th }}-29^{\text {th }}$ December-07 on topic "Face Recognition method 
based on Principle Component Analysis (PCA) \& Feed Forward Neural Network". The article number is 89 in Proceeding in the category Image Processing on the page number-422-424.

8. National Conference on Electronics, Communication \& Automation (NCECA) @ COE, Pandharpur on $25^{\text {th }} \& 26^{\text {th }}$ December 2008 on "Face Recognition method based on Principle Component Analysis (PCA) \& Feed Forward Neural Network". The article number is 5 in Proceeding in the category Image Processing on the page number-16-18.

9. National Conference on Engineering, Technology \& Architecture@ D.Y. Patil COE, Kolhapur on $25^{\text {th }} \& 26^{\text {th }}$ December 2010 on "Face Recognition method based on Principle Component Analysis (PCA) \& Feed Forward Neural Network". Published in the Proceeding in the category Computer Science Engineering on the page number-250-253. 